\title{
VENTILACIÓN NO INVASIVA EN EL PACIENTE CON FALLA RESPIRATORIA AGUDA
}

\author{
NONINVASIVE VENTILATION IN ACUTE PEDIATRIC RESPIRATORY FAILURE
}

\section{Dra. Leticia Yáñez P.}

Pediatra Intensivista, Médico Jefe Unidad de Paciente Crítico Pediátrico Clínica Santa María.

Profesor Universidad de Los Andes.

\begin{abstract}
Although most cases of respiratory failure resolve with medical treatment and are self-limited, some of them may require a higher level of ventilatory support.

Noninvasive ventilation (NIV) is an alternative to improve gas exchange in selected patients and may prevent intubation, the use of invasive ventilation and its associated risks. This article describes the use of noninvasive ventilation in acute respiratory pathology.

Keywords: non invasive ventilation, positive pressure, mask
\end{abstract}

RESUMEN

Aunque la mayoría de los casos de falla respiratoria son de evolución favorable y autolimitados algunos pueden requerir soporte ventilatorio de mayor nivel.

La ventilación no invasiva (VNI) es una alternativa que mejora el intercambio gaseoso en pacientes seleccionados y puede prevenir la intubación y uso de ventilación invasiva con sus riesgos asociados. Este artículo describe el uso de la ventilación no invasiva en la patología respiratoria aguda.

Palabras clave: ventilación no invasiva, presión positiva, máscara

\section{INTRODUCCIÓN}

La insuficiencia respiratoria aguda es originada por un desequilibrio entre la demanda y la capacidad de respuesta del sistema respiratorio, que por distintas causas no puede adaptar su rendimiento a las variaciones de la demanda metabólica. Para vencer las consecuencias de una patología respiratoria aguda, que pudiera interferir con el intercambio gaseoso, el sistema respiratorio responde con un aumento del volumen corriente $y / 0$ la frecuencia respiratoria.

Si la función de los músculos respiratorios es insuficiente para mantener un nivel de intercambio gaseoso a nivel alveolar que se adecúe a las necesidades metabólicas de la respiración celular, entonces resulta una insuficiencia respiratoria. Esta es una causa importante de morbilidad en Pediatría en relación con patologías pulmonares y de vía aérea, pero también puede desencadenarse por alteraciones de los sistemas nerviosos central y periférico y el sistema musculoesquelético (1).

En general la falla respiratoria se clasifica en dos grupos:

- Tipo I ó hipoxémica: la principal alteración es a nivel de relación ventilación perfusión $(\mathrm{V} / \mathrm{Q})$, es la más frecuente y se relaciona con la mayoría de las enfermedades pulmonares

\section{Correspondencia:}

Dra. Leticia Yáñez

Unidad Paciente Crítico Pediátrico

Clinica Santa Maria

Av Santa María 0500, Providencia

Santiago de Chile

Correo electrónico: Iyanez@clinicasantamaria.cl agudas pediátricas. Caracterizada por una $\mathrm{PaO}_{2}<60 \mathrm{~mm} \mathrm{Hg}$ con una $\mathrm{PaCO}_{2}$ normal o baja. Su tratamiento primario es la administración de oxígeno a un nivel suficiente para aumentar la saturación arterial de $\mathrm{O}_{2}\left(\mathrm{SpO}_{2}\right)$ a $>94 \%$. Si se requiere una fracción inspirada de $\mathrm{O}_{2}\left(\mathrm{FiO}_{2}\right)>0.5$ para alcanzar esta meta se define como falla respiratoria aguda hipoxémica.

- Tipo II o hipercápnica: es una consecuencia de la falla ventilatoria, que puede ocurrir en condiciones que afectan a la bomba respiratoria como alteraciones de tronco cerebral, neuromusculares y obstrucción aguda o crónica de la vía aérea superior. Se caracteriza por hipoventilación alveolar ó $\mathrm{PaCO}_{2}>$ $50 \mathrm{mmHg}$. Su desarrollo puede ser insidioso en patologías agudas como neumonía o asma, en las cuales a la alteración V/Q se agrega fatiga de los músculos respiratorios, sumándose a la hipoxemia una hipercapnia. La administración de oxigeno en la falla tipo II crónica puede ser riesgosa, ya que en casos de hipercapnia crónica, la hipoxemia es el único gatillante de los quimiorreceptores periféricos sensibles a oxigeno para mantener el control respiratorio. Por lo cual a la terapia con oxigeno suplementario debiera agregarse un apoyo para reducir la carga muscular respiratoria y la ventilación alveolar.

\section{VENTILACIÓN NO INVASIVA}

La ventilación no invasiva (VNI) puede ser una alternativa que mejora el intercambio gaseoso en pacientes seleccionados y puede prevenir la intubación y uso de ventilación invasiva con sus riesgos asociados, tales como trauma de la vía aérea superior e infecciones nosocomiales.

Se define como la entrega de presión positiva, 
continua 0 intermitente a través de una interfase sin necesidad de un tubo endotraqueal o cánula de traqueostomia. Puede ser una presión positiva continua sobre la vía aérea (CPAP) 0 un binivel de soporte respiratorio que incluye una presión positiva espiratoria (EPAP) y una presión positiva inspiratoria (IPAP) la cual es gatillada por el esfuerzo inspiratorio espontáneo del paciente. Dichas presiones son entregadas a través de cánulas nasales, máscaras faciales o cascos. Presenta gran flexibilidad en su aplicación y remoción y preserva los mecanismos de defensa de la vía aérea.

Desde el punto de vista fisiopatológico en una patología respiratoria la inflamación de la vía aérea, la ocupación y el colapso alveolar favorecen una alteración de la relación $V / Q$, efecto shunt, atrapamiento aéreo que determinarán una disminución de la compliance del sistema respiratorio y aumento de la resistencia de la vía aérea, hipoxemia y aumento del trabajo respiratorio que puede llevar a la fatiga de los músculos respiratorios, con hipoxemia e incluso hipercapnia secundaria. La VNI puede mejorar el reclutamiento alveolar, disminuir el trabajo respiratorio y por ende la fatiga muscular, contribuyendo a evitar la intubación (1).

Recientemente la Conferencia Consenso de Daño Pulmonar Agudo Pediátrico, luego de revisar la literatura disponible sobre $\mathrm{VNI}$ en relación con Sindrome de Distress
Respiratorio Agudo, de acuerdo a la definición de Berlin (2) concluye que su uso puede ser beneficioso, mejorar el intercambio gaseoso y potencialmente evitar la intubación, especialmente en niños con SDRA leve. Resaltan la necesidad de su aplicación en forma monitorizada en una unidad de cuidados críticos al paciente adecuado y con personal especializado debido a que es necesario estar atentos a los signos y síntomas de compromiso progresivo de la enfermedad que motivó su uso, eventuales complicaciones, sincronía paciente ventilador y necesidad de intubación por falta de respuesta a la terapia (3).

En 2008 realizamos un estudio en dos unidades de paciente crítico de Santiago de Chile, prospectivo randomizado en niños de 1 mes a 15 años con patología respiratoria aguda, entre un grupo control y un grupo con uso de binivel de presiones, con un protocolo bien definido de aplicación de VNI basado en un puntaje de dificultad respiratoria, requerimientos de oxigeno,y falla del método, determinó una disminución de la frecuencia cardiaca y respiratoria y una disminución de la necesidad de intubación y conexión a ventilación mecánica en forma estadísticamente significativa ( $28 \%$ de los pacientes en Bipap versus $68 \%$ de intubación de los casos control) (4). El protocolo utilizado en este estudio se adjunta en la Tabla № 1.

\begin{tabular}{|c|c|}
\hline Criterios de ingreso & $\begin{array}{ll}\text { - } & \text { Patología respiratoria aguda } \\
\text { - } & \text { Requerimientos de } \mathrm{O}_{2}>35 \% \text { para saturar } \geq 94 \% \\
\text { - } & \text { Dificultad respiratoria moderada según scores } \\
\text { - } & \text { Estabilidad hemodinámica }\end{array}$ \\
\hline Criterios de exclusión & $\begin{array}{l}\text { - Incapacidad de proteger las vías aéreas en pacientes en coma, Glasgow } \leq 8 \text { o con compromiso de tronco cerebral } \\
\text { - } \\
\text { - } \quad \text { Dificultad para aplicar la interfase como en trauma o quemadura facial o anormalidades craneofaciales. } \\
\text { - Compromiso gastrointestinal severo: hiperemesis, distensión abdominal, obstrucción intestinal o cirugía abdominal reciente. } \\
\text { - } \quad \text { Hipoxemia refractaria: sat }<90 \% / \mathrm{FiO}_{2}>=60 \% \\
\text { - } \quad \text { Exceso de secreciones traqueobronquiales } \\
\text { - } \quad \text { Intolerancia a la interface, agitación psicomotora } \\
\text { - } \quad \text { Falta de disponibilidad de personal entrenado en VNI y monitorización adecuada }\end{array}$ \\
\hline Aplicación de protocolo & 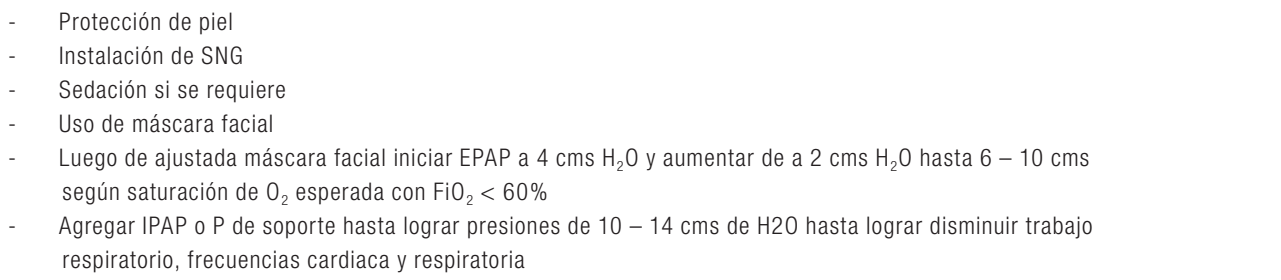 \\
\hline Criterios de mejoría & $\begin{array}{l}\text { - Persistencia de dificultad respiratoria moderada a severa a pesar de no filtración de la interfase y de lograrse } \\
\text { las presiones adecuadas. Falta de sincronización paciente/VNI por agitación a pesar de sedación. Considerar } \\
\text { intubación y uso de VM invasiva. No retrasar la decisión. } \\
\text { - Disminución progresiva de requerimientos de presiones, que se bajarán cuando se logre disminuir } \mathrm{FiO}_{2} \text { a }<50 \% \\
\text { - Se disminuirá IPAP en } 1-2 \mathrm{~cm} \text { según clínica (mejoría de expansión pulmonar y disminución de trabajo respiratorio) } \\
0 \text { manteniendo Vt } \geq 6 \mathrm{ml} / \mathrm{kg} \text { hasta suspensión de IPAP. } \\
\mathrm{FiO}_{2}<40 \% \text { para saturar }>93 \% \text { luego de suspensión de IPAP y con disminución progresiva de EPAP a } 6 \text { cms H2O }\end{array}$ \\
\hline
\end{tabular}

Tabla 1. Protocolo uso Ventilación No Invasiva en falla respiratoria aguda 
En un estudio multicéntrico realizado en 60 unidades pediátricas de Latinoamérica, España y Portugal en un periodo de un mes de infecciones respiratorias ingresaron 2156 pacientes, $42 \%$ presentaban falla respiratoria aguda, $75 \%$ de las cuales se debieron a patología respiratoria aguda: Neumonia, bronquiolitis, obstrucción de la via aérea superior, síndrome de distress respiratorio agudo 0 aspiración. En 155 pacientes se utilizó ventilación no invasiva, con un $39 \%$ de falla y necesidad de apoyo invasivo (5). Un estudio observacional prospectivo reciente, de dos años de duración describe uso de VNI en su mayoría en pacientes con patología respiratoria aguda, con un $30 \%$ de necesidad de intubación (6).

En la literatura se describen diversos factores que es necesario tener presentes al momento de definir a que pacientes beneficiaremos y no retardaremos la intubación agregando morbimortalidad. Entre los factores predictores de falla de VNI en Pediatría se describen:

- Inadecuada selección de pacientes: la presencia de una enfermedad severa, dada por un score de gravedad mayor, compromiso de mas de dos órganos (7).

- SDRA $(8,9)$.

- Tiempos de evaluación de respuesta a VNI según saturación y dificultad respiratoria: relación $\mathrm{SpO}_{2} /$ $\mathrm{FiO}_{2}<193$ después de 1 hora de VNI (9), $\mathrm{FiO}_{2}>80 \%$ después de 1 hora en NIV (10), falta de mejoría de la frecuencia respiratoria después de 2 horas de VNI (8) 0 después de 6 horas (9).

- Necesidad de presiones elevadas y de $\mathrm{FiO}_{2}>60 \%$ : un nivel de presión de soporte alto, $\mathrm{P}$ promedio $>12 \mathrm{~cm}$ $\mathrm{H}_{2} \mathrm{O} \circ \mathrm{FiO}_{2}>$ 0.6.(11).

\section{DISPOSITIVOS}

En la unidad de cuidados intensivos pediátricos se utilizan ventiladores diseñados para apoyo no invasivo y también algunos ventiladores invasivos que ofrecen una opción de VNI. En ambos casos puede haber una limitación para su uso en lactantes menores de 6 meses o de $10 \mathrm{~kg}$ debido a los sistemas de compensación de fuga insuficientes o de gatillo inapropiado. Los ventiladores de nueva generación son capaces de superar estos problemas; mientras que los ventiladores no invasivos pueden manejar las fugas y permitir un ajuste mas acotado de la sensibilidad y una mejor sincronía paciente ventilador que los primeros $(12,13)$.

\section{INTERFASES}

Es el dispositivo a través del cual el paciente se conecta a la tubuladura del ventilador, con la consecuente entrega de gas presurizado. Se encuentran disponibles de distintas formas, tamaños y materiales. La tolerancia del paciente y por lo tanto la efectividad de la VNI, dependerá de que no cause incomodidad, no genere lesiones dérmicas o escape aéreo significativo. Deben ser transparentes para permitir la adecuada monitorización del paciente. No existen estudios pediátricos que definan cual es la más apropiada en el paciente crítico. La elección se basará en la experiencia del equipo médico y en la edad y peso del paciente.
En lactantes se usarían más las interfases nasales mientras que en niños mayores serían mejor toleradas aquellas oronasales.

\section{SINCRONIZACIÓN}

La relación entre el apoyo ventilatorio no invasivo y el esfuerzo respiratorio espontáneo del niño es relevante en el éxito de la VNI (14). Existen distintos tipos de asincronia que se pueden clasificar en de gatillo, entrega y tiempo de flujo. La más frecuente en niños es el autogatillado que es la entrega de una ventilación no gatillada por el paciente, generalmente debido a fuga a través de la interfase y que da cuenta de la importancia de ellas y de la capacidad del ventilador de compensar fugas.

Basados en la literatura actual podemos afirmar que la VNI es una herramienta útil en el manejo de la falla respiratoria aguda, en pacientes pediátricos, aplicada bajo protocolos bien establecidos, por personal calificado y con monitorización adecuada, ya que es muy importante pesquisar en forma precoz, antes de 6 - 12 horas a aquellos pacientes que requerirán conexión a VM (9).

A continuación revisaremos su uso en las patologías respiratorias agudas más frecuentemente ingresadas a una unidad de paciente crítico pediátrico.

\section{VENTILACIÓN NO INVASIVA Y BRONQUIOLITIS AGUDA POR VIRUS RESPIRATORIO SINCICIAL (VRS)}

La bronquiolitis por VRS es la causa más frecuente de dificultad respiratoria en lactantes $<1$ año, y representa el $2-6 \%$ de los ingresos a unidades pediátricas de paciente crítico (UPCP). Se caracteriza por un estrechamiento severo de la vía aérea periférica generando obstrucción severa y aumento de la resistencia de la vía aérea. En espiración se genera una disminución desproporcionada del flujo debido al colapso dinámico de la vía aérea desencadenando hiperinsuflación dinámica y PEEP intrínseco (PEEPi). Los efectos de esta alteración serán aumento hasta en 6 veces del trabajo muscular necesario para iniciar el flujo inspiratorio, ya que se requiere mayor presión pleural negativa para movilizar el aire hacia un alveolo con presión positiva, haciendo a los músculos inspiratorios relativamente menos eficientes. El patrón respiratorio presenta una frecuencia respiratoria aumentada, disminución del volumen corriente y la presión inspiratoria, una disminución del tiempo espiratorio y un aumento en la relación Ti/T respiratorio total, con la consecuente disminución del tiempo de eliminación del volumen y mayor atrapamiento aéreo. En niños, la proporción de fibras tipo 1 del diafragma es reducida, generando una menor resistencia del músculo respiratorio a la sobrecarga, haciendo al paciente pediátrico más propenso a la fatiga muscular. Si la energía necesaria para superar la resistencia de la vía aérea y la retracción elástica exceden la capacidad de los músculos respiratorios de asegurar una ventilación adecuada, entonces se genera hipercapnia y la necesidad de apoyo ventilatorio.

La mantención de una presión continua durante el ciclo respiratorio completo, con CPAP nasal mejora el trabajo respiratorio en lactantes de $<3$ y 6 meses, al disminuir el esfuerzo inspiratorio necesario para superar el PEEPi, con una 
disminución del colapso espiratorio de la vía aérea superior e inferior, favoreciendo el flujo espiratorio, como lo han demostrado distintos estudios publicados recientemente $(15,16)$. En estos casos se usó como criterio de inclusión pacientes con bronquiolitis por VRS, con $\mathrm{pPCO}_{2}>50 \mathrm{mmHg}$ y un puntaje de asma clínica de Wood modificado, $>5$ (Tabla 2). Se realizaron mediciones de presiones esofágicas y gástricas para evaluar la carga muscular respiratoria. Se aplicó un CPAP de $6 \mathrm{~cm} \mathrm{H}_{2} \mathrm{O}$, a través de un Infant Flow Ventilator (Electro Medical Equipment, UK) con sistema de humidificación. Los efectos fueron observados desde la primera hora de CPAP nasal y se mantuvieron a las 6 horas. En 2011, Essouri y cols encontraron en 10 lactantes con falla respiratoria hipercápnica debida a bronquiolitis aguda, que el nivel de CPAP nasal que mas disminuía el trabajo respiratorio y mejoraba el patrón respiratorio era de $7 \mathrm{~cm}$ de $\mathrm{H}_{2} \mathrm{O}$ (17). En un estudio de cohorte realizado en dos centros europeos, se encontró que de 135 lactantes menores de 6 meses, con enfermedad de la via respiratoria baja por VRS, 89 se conectaron a CPAP nasal y 35 a VM invasiva. En esta serie los pacientes en VNI tuvieron menos días de UCI y apoyo ventilatorio, sin embargo tuvieron diferencias significativas en cuanto a menores puntajes de gravedad y mayor $\mathrm{SpO}_{2} / \mathrm{FiO}_{2}$ que al ingresar. Sugieren la necesidad de estudios prospectivos randomizados para recomendar CPAP en bronquiolitis (18). En lactantes entre 6 y 12 meses con bronquiolitis y $\mathrm{PF}<300$ se comparó administración de CPAP entre 4 y $10 \mathrm{~cm}$ de agua a través de casco y mascara facial. Ninguno requirió intubación, y se encontraron diferencias significativas en cuanto a mejor tolerancia y menor necesidad de sedación en pacientes con uso de cascos que con mascara facial (19). La utilidad del apoyo VNI sería mejorar el esfuerzo muscular respiratorio, evitar colapso alveolar y así evitar progresión a agotamiento. En casos de bronquiolitis por VRS se ha usado binivel de presiones a través de máscara facial, con IPAP entre 10 - 14 y EPAP 6 - 10 demostrando disminución de la dificultad respiratoria y de la necesidad de intubación (4). En una serie retrospectiva se usó CPAP y Bipap en pacientes con bronquiolitis y la falla del método se relacionó con sobreinfección bacteriana, prematuridad y cardiopatías (20). Fisiológicamente la presión inspiratoria ayuda a apoyar la musculatura respiratoria fatigada y por lo tanto disminuye la disnea y mejora el intercambio gaseoso.

\begin{tabular}{|l|c|c|c|c|}
\hline & 0 & 0.5 & 1 & 2 \\
\hline Cianosis & $\begin{array}{c}\mathrm{SpO}_{2} \geq 95 \% \mathrm{con} \\
\mathrm{FiO}_{2} 0.21\end{array}$ & $\begin{array}{c}95 \%>\mathrm{SpO}_{2} \geq \\
9 \% \mathrm{con} \mathrm{FiO}_{2} \\
0.21\end{array}$ & $\begin{array}{c}\mathrm{SpO}_{2} \geq 90 \% \mathrm{con} \\
\mathrm{FiO}>0.21\end{array}$ & $\begin{array}{c}\mathrm{SpO}_{2}<90 \% \mathrm{con} \\
\mathrm{FiO}_{2}>0.21\end{array}$ \\
\hline Ruidos inspiratorios & Normales & Disminuidos & Disminuidos & $\begin{array}{c}\text { Disminuidos 0 } \\
\text { ausentes }\end{array}$ \\
\hline Uso musculatura accesoria & VAS $<5 \mathrm{~cm}$ & $10 \geq$ VAS $>5 \mathrm{~cm}$ & 15 VAS $>10 \mathrm{~cm}$ & VAS $\geq 15 \mathrm{~cm}$ \\
\hline Ruidos espiratorios & VAS $<5 \mathrm{~cm}$ & $10 \geq$ VAS $>5 \mathrm{~cm}$ & 15 VAS $>10 \mathrm{~cm}$ & VAS $\geq 15 \mathrm{~cm}$ \\
\hline Compromiso sensorio & Normal & Agitado al estímulo & Deprimido 0 agitado & Coma \\
\hline
\end{tabular}

Tabla 2. Score clínico de asma de Wood modificado

\section{VENTILACIÓN NO INVASIVA Y SÍNDROME DE DIFICULTAD RESPIRATORIA AGUDO (SDRA) PEDIÁTRICO}

En SDRA se observa daño endotelial capilar con aumento de permeabilidad y daño del epitelio alveolar, con inflamación y trastorno del drenaje de fluidos con o sin disfunción del surfactante pulmonar. Se encuentra inflamación de la vía aérea, ocupación y colapso alveolar. Como consecuencia se produce alteración del intercambio gaseoso. El SDRA es una enfermedad heterogénea que puede ser de causa pulmonar 0 extrapulmonar, que genera hipoxemia debido a la alteración de la relación ventilación/perfusión, aumento del espacio muerto y atrapamiento aéreo. En niños observamos una disminución de la capacidad residual funcional y de la compliance y aumento de la resistencia de la vía aérea. El trabajo respiratorio aumenta y por lo tanto aumenta la demanda de oxígeno de los músculos respiratorios, agravando la hipoxemia e incluso determinando fatiga muscular y retención de $\mathrm{CO}_{2}$.

La implementación de VNI, (CPAP y Binivel de presiones) reduce el trabajo muscular respiratorio, facilita la apertura de la vía aérea y mejora la oxigenación (1). Estudios fisiológicos y clínicos sugieren que el apoyo adicional 
inspiratorio, a través de máscara facial, mejora la oxigenación y la ventilación al descargar los músculos inspiratorios (1, 4, 21). Si bien la literatura pediátrica dispone a la fecha de pocos estudios prospectivos, randomizados que apoyen el uso de VNI en SDRA, el Consenso Pediátrico, luego de hacer una revisión de la literatura al respecto sugiere su uso en pacientes con SDRA leve a moderado (3).

\section{VENTILACIÓN NO INVASIVA Y ESTADO ASMÁTICO}

Una patología frecuente en la UPCP, especialmente en los meses de primavera o de invierno, es el ingreso de pacientes con antecedentes de asma que han suspendido su tratamiento y/o que presentan un cuadro infeccioso viral intercurrente que los descompensa, con signología bronquial obstructiva severa.

Se define asma aguda severa o estado asmático como una exacerbación severa y rápida de un asma que puede no responder al tratamiento con oxígenos, broncodilatadores y corticoides sistémicos. Se caracteriza por una obstrucción de la vía aérea baja debido a inflamación, edema, broncoespasmo y tapones mucosos. Presentan limitación al flujo aéreo y cierre prematuro de la vía aérea con aumento del trabajo respiratorio, con aumento de la resistencia de la vía aérea e hiperinsuflación con sobredistensión pulmonar, atrapamiento aéreo y PEEP intrínseco. La fase espiratoria se hace activa para poder vaciar los alveolos.

La VNI en estos casos, tendría un efecto broncodilatador con reclutamiento alveolar, resultante de el uso de PEEP que contrarresta el PEEP intrínseco y favorece la apertura de la vía aérea colapsada disminuyendo el esfuerzo necesario para generar una presión negativa que favorezca el flujo inspiratorio de aire. La VNI desplaza hacia distal el punto de igual presión, que es uno de los mecanismos fisiopatológicos básicos de porque funciona la VNI en paciente obstructivo. El flujo aéreo aumenta a través de los canales ventilatorios colaterales favoreciendo la reexpansión de áreas colapsadas y mejoría de la relación $\mathrm{V} / \mathrm{Q}$. La aplicación de una presión inspiratoria ayuda a disminuir el esfuerzo muscular respiratorio y aumenta el volumen corriente.

En un análisis de artículos de estado asmático y VNI, dos trabajos prospectivos observacionales, un reporte de caso y dos estudios retrospectivos de aplicación de Bipap a través de mascaras nasales o faciales en niños de 2 a 21 años que no responden al manejo convencional de crisis asmáticas, evidencian buena tolerancia al tratamiento no invasivo, disminución del trabajo respiratorio y mejoría de la oxigenación, con complicaciones como enfisema subcutáneo o neumotórax en $1 \%(22)$. En esta revisión también se describe un trabajo prospectivo, randomizado en 20 pacientes de 1 a 20 años con tratamiento habitual y Bipap para alcanzar volumen corriente de 6 a $8 \mathrm{ml} / \mathrm{kg}$, por máscara nasal o facial en el que se encontró buena tolerancia al método, mejoría del puntaje de severidad de asma y disminución de los requerimientos de oxígeno, sin complicaciones mayores ni requerimientos de intubación (23). En nuestra experiencia usamos Bipap en pacientes con crisis asmática que ingresan con requerimientos de oxígeno mayores a $35 \%$, trabajo respiratorio aumentado, sin necesidad de intubación en ninguno de ellos (4).

\section{CONCLUSIÓN}

La VNI ha aumentado durante las últimas dos décadas. Si bien faltan trabajos prospectivos randomizados la experiencia acumulada permite afirmar que es un método seguro en pacientes con patología aguda con insuficiencia respiratoria moderada. Debe ser aplicada en una unidad con personal capacitado, con monitorización continua que permita pesquisar precozmente a aquellos pacientes que no respondieron para no retrasar su intubación y conexión a VM invasiva.

\section{La autora declara no presentar conflicto de interés.}

\section{REFERENCIAS}

1. L'Her E, Deye N, Lellouche F, Taille S, Demoule A, Fraticelli A, Mancebo $J$, Brochard L. Physiologic effects of noninvasive ventilation during acute lung injury. Am J Respir Crit Care Med 2005; 172 (9):1112-1118.

2. The ARDS Definition Task Force. Acute Respiratory Distress Syndrome: The Berlin Definition, JAMA 2012; 307 (23): 2526 - 2533.

3. Essouri S, MD, PhD1; Carroll C, MD, MS2; for the Pediatric Acute Lung Injury Consensus Conference Group. Noninvasive Support and Ventilation for Pediatric Acute Respiratory Distress Syndrome: Proceedings From the Pediatric Acute Lung Injury Consensus Conference. Pediatr Crit Care Med 2015; 16 (5 Suppl 1): S102 - 110.

4. Yanez L, Yunge $M$, Emilfork $M$, Lapadula $M$, Alcántara $A$, Fernández $\mathrm{C}$, et al. A prospective, randomized, controlled trial of noninvasive ventilation in pediatric acute respiratory failure. Pediatr Crit Care Med 2008; 9(5): $484-489$.

5. Farías J, Fernández A, Monteverde E, Flores JC, Baltodano A, Menchaca $A$ et al. Mechanical ventilation in pediatric intensive care units during the season for acute lower respiratory infection: A multicenter study*. Pediatr Crit Care Med 2012; 13:158-164.

6. Yaman A, Kendirli, Odek C, Ates C, Tasyapar N, Ince E, et al. Efficacy of noninvasive mechanical ventilation in prevention of intubation and reintubation in the pediatric intensive care unit. J Crit Care. 2016;32:175 $-181$.

7. Piastra M, De Luca D, Marzano L, Stival E, Genovese 0, Pietrini D, et al. The number of failing organs predicts non- invasive ventilation failure in children with ALI/ARDS. Intensive Care Med 2011; 37: 1510-6.

8. Essouri S, Chevret L, Durand P, Haas V, Fauroux B, Devictor D. Noninvasive positive pressure ventilation: five years of experience in a pediatric intensive care unit. Pediatr Crit Care Med 2006; 7: 329-34.

9. Mayordomo-Colunga J, Pons M, López Y, Solana MJ, Rey C, MartínezCamblor $\mathrm{P}$ et al. Predicting non-invasive ventilation failure in children from the Sp02/Fi02 (SF) ratio. Intensive Care Med 2013; 39:1095-1103. 10. Bernet V, Hug MI, Frey B. Predictive factors for the success of noninvasive mask ventilation in infants and children with acute respiratory failure. Pediatr Crit Care Med 2005; 6: 660-4.

11. Munoz-Bonet JI, Flor-Macian EM, Brines J, Rosello-Millet PM, Cruz Llopis M, Lopez-Prats JL, et al. Predictive factors for the outcome of noninvasive ventilation in pediatric acute respiratory failure. Pediatr Crit Care Med 2010; 11: 675-80.

12. Rimensberger PC, Heulitt MJ, Meliones J, Pons M, Bronicki RA. Mechanical ventilation in the pediatric cardiac intensive care unit: the essentials. World J Pediatr Congenit Heart Surg 2011; 2: 609-19.

13. Fauroux B, Leroux K, Desmarais G, Isabey D, Clement A, Lofaso 
$F$ et al. Performance of ventilators for noninvasive positive-pressure ventilation in children. Eur Respir J 2008; 31: 1300-7.

14. Carteaux G, Lyazidi A, Cordoba-Izquierdo A, Vignaux L, Jolliet P, Thille AW, et al. Patient-ventilator asynchrony during noninvasive ventilation: a bench and clinical study. Chest 2012; 142: 367-76.

15. Milési $C$, Matecki $S$, Jaber $S$, Mura $T$, Jacquot $A$, Pidoux 0 et al. $6 \mathrm{cmH} 2 \mathrm{O}$ Continuous Positive Airway Pressure Versus Conventional Oxygen Therapy in Severe Viral Bronchiolitis: A Randomized Trial. Pediatr Pulmonol. 2013; 48:45-51.

16. Cambonie G, Milési C, Jaber S, Amsallem F, Barbotte E, Picaud JC et al. Nasal continuous positive airway pressure decreases respiratory muscles overload in young infants with severe acute viral bronchiolitis. Intensive Care Med 2008; 34:1865-1872.

17. Essouri S, Durand P, Chevret L, Balu L, Devictor D, Fauroux B, Tissières $P$. Optimal level of nasal continuous positive airway pressure in severe viral bronchiolitis. Intensive Care Med 2011; 37:2002-2007

18. Borckink I, Essouri S, Laurent M, Albers M, Burgerhof J, Tissières $P$. Infants with severe respiratory syncytial virus needed less ventilator time with nasal continuous airways pressure then invasive mechanical ventilation. Acta Pediátrica 2014; 103: 81-85.

19. Chidni G, Piastra M, Marchesi T, De Luca D, Napolitano L, Salvo I et al. Continuous Positive Airway Pressure With Helmet Versus Mask in Infants With Bronchiolitis: An RCT. Pediatr 2015;135;e868 - 75.

20. Javouhey E, Barats A, Richard N, Stamm D, Floret D. Non-invasive ventilation as primary ventilatory support for infants with severe bronchiolitis. Intensive Care Med 2008; 34:1608-1614.

21. Zhan Q, Sun B, Liang L, Yan X, Zhang L, Yang $J$ et al: Early use of noninvasive positive pressure ventilation for acute lung injury: $A$ multicenter randomized controlled trial. Crit Care Med 2012; 40:455460.

22. de Souza P, Saldanha S, Barreto M. Noninvasive ventilation in status asthmaticus in children: levels of evidence. Rev Bras Ter Intensiva. 2015;27(4):390-396.

23. Basnet S, Mander G, Andoh J, Klaska H, Verhulst S, Koirala J. Safety, efficacy, and tolerability of early initiation of noninvasive positive pressure ventilation in pediatric patients admitted with status asthmaticus: a pilot study. Pediatr Crit Care Med. 2012;13(4):393- 398. 